This is an electronic reprint of the original article. This reprint may differ from the original in pagination and typographic detail.

Author(s): Kauppila, Ville J.; Hyart, Timo; Heikkilä, Tero

Title: $\quad$ Collective amplitude mode fluctuations in a flat band superconductor formed at a semimetal surface

Year: $\quad 2016$

Version:

Please cite the original version:

Kauppila, V. J., Hyart, T., \& Heikkilä, T. (2016). Collective amplitude mode fluctuations in a flat band superconductor formed at a semimetal surface. Physical Review B, 93(2), Article 024505. https://doi.org/10.1103/PhysRevB.93.024505

All material supplied via JYX is protected by copyright and other intellectual property rights, and duplication or sale of all or part of any of the repository collections is not permitted, except that material may be duplicated by you for your research use or educational purposes in electronic or print form. You must obtain permission for any other use. Electronic or print copies may not be offered, whether for sale or otherwise to anyone who is not an authorised user. 


\title{
Collective amplitude mode fluctuations in a flat band superconductor formed at a semimetal surface
}

\author{
V. J. Kauppila, ${ }^{1}$ T. Hyart, ${ }^{2}$ and T. T. Heikkilä ${ }^{2}$ \\ ${ }^{1}$ O.V. Lounasmaa Laboratory, Aalto University, P.O. Box 15100, FI-00076 AALTO, Finland* \\ ${ }^{2}$ University of Jyvaskyla, Department of Physics and Nanoscience Center, \\ P.O. Box 35 (YFL), FI-40014 University of Jyväskylä, Finland
}

(Dated: October 7, 2015)

\begin{abstract}
We study the fluctuations of the amplitude (i.e. the Higgs-Anderson) mode in a superconducting system of coupled Dirac particles proposed as a model for possible surface or interface superconductivity in rhombohedral graphite. This system also serves as a generic model of a topological semimetal with an interaction driven transition on its surface. We show that the absence of Fermi energy and vanishing of the excitation gap of the collective amplitude mode in the model leads to a large fluctuation contribution to thermodynamic quantities such as the heat capacity. As a consequence, the mean-field theory becomes inaccurate indicating that the interactions lead to a strongly correlated state. We also present a microscopic derivation of the Ginzburg-Landau theory corresponding to this model.
\end{abstract}

A topological flat band spectrum can emerge as a surface or interface state of topological semimetals ${ }^{1,2}$. Such a spectrum has been proposed to occur in various semimetals, such as rhombohedrally stacked graphite ${ }^{3-5}$, at dislocation interfaces in bernal stacked graphite ${ }^{6}$, edges of zig-zag graphene ${ }^{7}$, three-dimensional honeycomb structures ${ }^{8}$, inversion-symmetric crystals ${ }^{9}$, antiperovskite $\mathrm{Cu}_{3} \mathrm{PdN}^{10}$, a polymorph of $\mathrm{Ca}_{3} \mathrm{P}_{2}{ }^{11}$ and three dimensional graphene networks ${ }^{12}$. A flat band can also take place at the interfaces between crystalline topological and non-topological insulators ${ }^{13}$. Such a state has a singular density of states which can, in the presence of an attractive pairing between the particles, lead to superconductivity with an unusually high critical temperature ${ }^{14,15}$. This mechanism has been suggested as an explanation to the unusually high critical temperature $T_{c}$ in some IV-VI semiconductor heterostructures ${ }^{16-18}$ and in various graphite based materials ${ }^{19-21}$. In the Hubbard model, related work has also been done to study superconductivity in the presence of a flat band ${ }^{22}$ and with Fermi energy close to van Hove singularities ${ }^{23}$.

We study here the effect of fluctuations on the superconducting properties of flat band superconductors in topological semimetals. We use the particular model proposed in Refs. 4 and 5 and also numerically analyzed in Ref. 24 for rhombohedrally stacked graphite. However, we argue that the form of the dispersion (1) and therefore most of the qualitative conclusions of this paper apply to all flat band surface states of topological semimetals. The model consists of $N$ individual graphene layers with $s$-wave pairing between the Dirac electrons coupled to form a stack of rhombohedral graphite. The flat band is formed on the surfaces of the stack where the superconductivity also appears (see Fig. 1). A peculiarity of this model is the closing of the gap in the fermionic excitation spectrum at the limit of a large number of layers even with an isotropic $s$-wave mean-field order parameter as can be seen from the surface state spectrum (see Fig.
$1)^{5}$

$$
E_{\mathbf{p}}^{2}=\left(1-\mathbf{p}^{2} / p_{\mathrm{FB}}^{2}\right)^{2}\left(\Delta_{0}^{2}+\xi_{\mathbf{p}}^{2}\right),
$$

where $\xi_{\mathbf{p}}=\gamma_{1}\left|\mathbf{p} / p_{\mathrm{FB}}\right|^{N}, \gamma_{1}$ is the interlayer coupling constant, $p_{\mathrm{FB}}=\gamma_{1} / v_{F}$ is the width of the flat band and $\Delta_{0}$ is the mean-field order parameter. (We use everywhere $\hbar=k_{B}=1$.) This expression is valid for $\left|\mathbf{p} / p_{\mathrm{FB}}\right|<1-1 / N .{ }^{25}$ The gap closes at the flat band edge along the line $|\mathbf{p}|=p_{\mathrm{FB}}$ in the bulk limit $N \rightarrow \infty$. This is due to the fact that there the surface states penetrate into the bulk and surface superconductivity cannot create a gap for the bulk excitations. This hence applies to all topological semimetals with a flat band surface state. Note that this spectrum is qualitatively different than, for example, in $d$-wave superconductors, where the gap in the fermionic spectrum vanishes in a set of points in the momentum space.

Even without making detailed calculations, simple arguments as for why fluctuations are expected to matter in this model can be made. Typically the magnitude of fluctuations is characterized by the Ginzburg number Gi $\sim 1-T^{*} / T_{c}$, which is related to the temperature $T^{*}$ above which the fluctuations dominate the thermodynamical properties. In a $2 \mathrm{D}$ BCS superconductor, the Ginzburg number is given by $G i \sim T_{c} / E_{F}$, where $E_{F}$ is the Fermi energy. For the flat band, $E_{F}=0$, leaving no other relevant energy scale available to be compared with $T_{c}$ which suggests ${ }^{5}$ that $G i \sim 1$. However, this argument is based on the analysis close to $T_{c}$ and it does not tell how low temperatures are needed to get rid of the effect of fluctuations. Below we show that due to the line node at $|\mathbf{p}|=p_{\mathrm{FB}}$ in the superconducting state, the fluctuation contribution is relevant down to $T=0$.

The aim of this paper is to show that in this model, the amplitude fluctuations provide a large contribution to thermodynamic observables (namely, the heat capacity). We show this both within the Ginzburg-Landau theory (close to $T_{c}$ ) and within a more general calculation based on functional integral formalism for the partition 


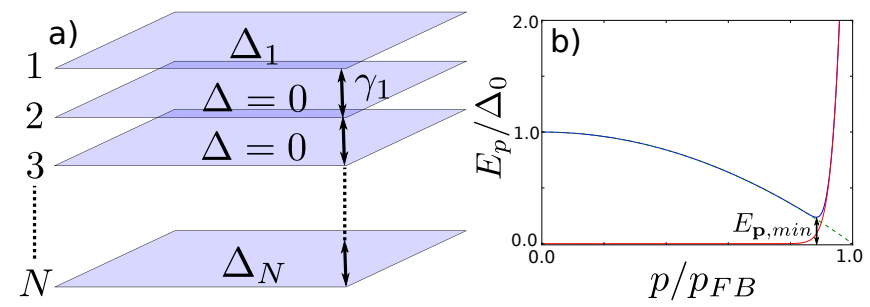

FIG. 1. (Color online) a) Schematic figure of the model which consists of $N$ parts with Dirac spectrum $H=v_{F} \sigma \cdot \mathbf{p}$ coupled via coupling strength $\gamma_{1}$. This results in an effective lowenergy theory described by the action (10). The superconductivity is localized at the surfaces where the Cooper pair field $^{26}$ is given by $\Delta_{1, N}$. b) Fermionic excitation spectrum of the surface state for the superconducting (blue line) and normal (red line) state. For finite $N$, the superconducting state has an excitation gap $E_{p, \min }$ which closes in the limit $N \rightarrow \infty$ (green dashed line).

function which preserves the fluctuations up to Gaussian order. At low temperatures, we find that the amplitude fluctuation contribution becomes equal to the fermionic contribution from the Bogoliubov quasiparticles.

A simple picture of the fluctuation contribution can be obtained from Ginzburg-Landau theory close to $T_{c}$. There, a superconductor is described in terms of the Cooper pair field $\Delta$ whose absolute value as well as the gradient are small so that an expansion with respect to them can be made. In the usual approach, only the value of the wave function that minimizes the GinzburgLandau free energy is considered, assuming that all the pairs are condensed to this state. The study of fluctuations in superconductors involves considering also Cooper pairs that are on some other states. In the field integral formalism we use below, this translates into taking an integral over different configurations of the Cooper pair fields and weighting different configurations by a factor that is given by the Ginzburg-Landau action when calculating observables.

From symmetry considerations, or from microscopic calculations (see below), we get for a general superconductor consisting of two separate symmetric parts (in our case, the top and the bottom layers of the graphite stack), a Ginzburg-Landau action of the form

$$
\begin{gathered}
S_{G L}=\int d^{2} x\left[\sum_{i \in\{1, N\}}\left(\alpha_{1}\left|\Delta_{i}\right|^{2}+\alpha_{2}\left|\nabla \Delta_{i}\right|^{2}+\beta\left|\Delta_{i}\right|^{4}\right)\right. \\
\left.-\gamma\left(\Delta_{1} \bar{\Delta}_{N}+\Delta_{N} \bar{\Delta}_{1}\right)\right]
\end{gathered}
$$

Here $\gamma$ is the Josephson coupling coefficient between the two parts characterized by amplitudes $\Delta_{1}$ and $\Delta_{N}$. $N$ in the index is the number of layers and we assume it to be large. The partition function, from which thermodynamic quantities can be calculated, is then given by

$$
\mathcal{Z}=\int\left(\prod_{i \in\{1, N\}} \mathcal{D} \Delta_{i} \mathcal{D} \bar{\Delta}_{i}\right) e^{-S_{G L}\left(\Delta_{i}, \bar{\Delta}_{i}\right)} .
$$

A microscopic calculation from the general action for our model, given in the next section, yields the following values for the Ginzburg-Landau coefficients:

$$
\begin{array}{cl}
\alpha_{1}=\frac{p_{\mathrm{FB}}^{2}}{48 \pi T_{\mathrm{C}}^{2}} \frac{T-T_{c}}{T_{c}}, & \alpha_{2}=\frac{1}{32 \pi T_{c}^{2}}, \\
\beta=\frac{p_{\mathrm{FB}}^{2}}{1920 \pi T_{c}^{4}}, & \gamma=\frac{\gamma_{1}^{2} p_{\mathrm{FB}}^{2}}{8 \pi T_{c}^{4} N^{5}} .
\end{array}
$$

From these coefficients one can extract a coherence length $\xi^{2} \sim \alpha_{2} /\left|\alpha_{1}\right|=3 T_{c} /\left(2 p_{\mathrm{FB}}^{2}\left|T_{c}-T\right|\right)$, which is very short $\left(\xi \sim v_{F} / \gamma_{1} \ll v_{F} / \Delta\right)$ for $T$ sufficiently below $T_{c}$ indicating the strong coupling nature of the superconductivity.

To consider fluctuations for $T<T_{c}$, we expand around the mean-field value of $\Delta_{0}^{2}=-\left(\alpha_{1}-\gamma\right) /(2 \beta)$ as $\Delta_{i}(x)=$ $\left(\Delta_{0}+\delta_{i}(x)\right) e^{i \phi_{i}(x)}$. The fluctuation part of the action can then be written in terms of four fluctuation modes:

$$
\begin{aligned}
& S_{1}=\int d^{2} x \frac{\alpha_{2}}{4 \beta}\left|\alpha_{1}-\gamma\right|\left(\nabla \phi_{+}\right)^{2} \\
& S_{2}=\int d^{2} x\left[\frac{\alpha_{2}}{2}\left(\nabla \delta_{+}\right)^{2}+\left|\alpha_{1}-\gamma\right| \delta_{+}^{2}\right] \\
& S_{3}=\int d^{2} x\left[\frac{\gamma\left|\alpha_{1}-\gamma\right|}{2 \beta} \phi_{-}^{2}+\frac{\alpha_{2}\left|\alpha_{1}-\gamma\right|}{4 \beta}\left(\nabla \phi_{-}\right)^{2}\right] \\
& S_{4}=\int d^{2} x\left[\frac{\alpha_{2}}{2}\left(\nabla \delta_{-}\right)^{2}+\left|\alpha_{1}-2 \gamma\right| \delta_{-}^{2}\right]
\end{aligned}
$$

so that $S_{G L}=S_{M F}+S_{1}+S_{2}+S_{3}+S_{4}$, where the mean-field part is given by $S_{M F}=-\left(\alpha_{1}-\gamma\right)^{2} /(2 \beta)$. The four fluctuation modes can be identified as the total phase (Nambu-Goldstone) mode $\left(S_{1}\right)$, the total amplitude (Higgs-Anderson) mode $\left(S_{2}\right)$, the relative phase (Leggett) mode $\left(S_{3}\right)$, and the relative amplitude mode $\left(S_{4}\right)$. In this new diagonal basis, the four independent fields are defined as $\phi_{ \pm}=\phi_{1} \pm \phi_{N}$ and $\delta_{ \pm}=\delta_{1} \pm \delta_{N}$. In the limit $N \rightarrow \infty$, the two phase and the two amplitude modes become identical and the action separates into two identical standard fluctuating Ginzburg-Landau actions with coefficients given by (4). The standard GinzburgLandau theory calculations can then be applied ${ }^{27}$ and we find that the total amplitude mode contribution to the heat capacity below $T_{c}$ becomes $^{28}$

$$
\delta C_{\text {amplitude }}=\frac{A p_{\mathrm{FB}}^{2} T_{c}}{3 \pi\left(T_{c}-T\right)} \sim \frac{\left|\alpha_{1}\right|}{\alpha_{2}} \frac{T_{c}^{2}}{\left(T_{c}-T\right)^{2}} A .
$$

Here $A$ is the surface area of the sample. In the lowest order in $T_{c}-T$, the phase mode does not contribute to the heat capacity. Setting $\delta C_{\text {amplitude }}\left(T^{*}\right)$ equal to the heat capacity jump at the transition,

$$
\Delta C=A p_{\mathrm{FB}}^{2} \frac{5}{6 \pi} \sim \frac{\alpha_{1}^{2}}{\beta} \frac{T_{c}^{2}}{\left(T-T_{c}\right)^{2}} A,
$$

and solving for temperature, $T^{*}$, yields the Ginzburg number

$$
G i \equiv \frac{T_{c}-T^{*}}{T_{c}}=\frac{2}{5} .
$$

This is of the order of unity as expected from purely dimensional arguments. While at the reduced temperature 
indicated by the Ginzburg number, the Ginzburg-Landau approach is not, strictly speaking, valid, this nevertheless gives us an estimate of the size of the fluctuations.

To understand the large fluctuations and compare them to conventional superconductors, we note that in both cases the fluctuation contribution and the heat capacity jump are proportional to the same Ginzburg-Landau coefficients, but the microscopic values of the coefficients differ greatly. In a conventional two-dimensional BCS superconductor ${ }^{27} \alpha_{1}=$ $N(0)\left(T-T_{c}\right) / T_{c}^{2}, \alpha_{2}=7 \zeta(3) N(0) v_{F}^{2} /\left(32 \pi^{2} T_{C}^{3}\right)$ and $\beta=7 \zeta(3) N(0) /\left(16 \pi^{2} T_{c}^{3}\right)$, where $\zeta(x)$ is the Riemann zeta function, $N(0)$ is the density of states at the Fermi level and $v_{F}$ is the Fermi velocity. There is one additional parameter compared to the case of the flat band model. This leads to different scales for the heat capacity jump and the fluctuation heat capacity in twodimensional BCS superconductors

$$
\delta C_{\text {amplitude }} \sim \frac{T_{c}}{E_{F}} \frac{T_{c}}{\left|T-T_{c}\right|} \Delta C
$$

resulting in a much smaller Ginzburg number $G i \sim$ $T_{c} / E_{F}$ compared to the flat band superconductors.

At low temperatures, the Ginzburg-Landau approach is invalid and we need a microscopic theory to account for the fluctuations. For the surface state, we can use the Bogoliubov - de Gennes equation derived in Ref. 4 to find that the action, after integrating out the Fermionic degrees of freedom, has the form

$$
\begin{gathered}
\mathcal{Z}=\int \mathcal{D} \Delta \mathcal{D} \bar{\Delta} \exp \left\{-\frac{V}{g T} \sum_{p}\left(\left|\Delta_{1, p}\right|^{2}+\left|\Delta_{N, p}\right|^{2}\right)\right. \\
\left.+\operatorname{tr} \ln \left[\frac{1}{T}\left(\begin{array}{cc}
\check{\Delta}_{1, p-p^{\prime}}-i \tilde{\omega}_{p} & \check{\tau}_{3} \xi_{\mathbf{p}} \\
\check{\tau}_{3} \xi_{\mathbf{p}} & \check{\Delta}_{N, p-p^{\prime}}-i \tilde{\omega}_{p}
\end{array}\right)\right]\right\} .
\end{gathered}
$$

Here $\xi_{\mathbf{p}}=\gamma_{1}\left(|\mathbf{p}| / p_{\mathrm{FB}}\right)^{N}, \tilde{\omega}_{p}=(2 n+1) \pi T /\left(1-|\mathbf{p}|^{2} / p_{\mathrm{FB}}^{2}\right)$ is the Matsubara frequency with a momentum dependent factor which comes from using the ansatz for surface state wave functions given in Ref. 4 , and $g$ is the superconducting coupling strength with dimensions of (energy) $\cdot$ (volume). The volume $V \equiv A d$, where $d \sim$ $1 / p_{\mathrm{FB}}$ is the spatial extent of the surface state wavefunctions. Here, $p \equiv(n, \mathbf{p})$ is the three-momentum which includes the two-dimensional momentum $\mathbf{p}$ in the plane of the layers and the Matsubara index $n$. The $4 \times 4$ matrix structure of the action (in addition to the momentum space degrees of freedom) results from top-bottom layer $\otimes$ particle-hole degrees of freedom. The Cooper pair fields in the trace are given by matrices

$$
\hat{\Delta}_{i, p-p^{\prime}}=\left(\begin{array}{cc}
0 & \Delta_{i, p-p^{\prime}} \\
\bar{\Delta}_{i, p^{\prime}-p} & 0
\end{array}\right)
$$

and can now have a non-zero momentum, i.e., $p-p^{\prime} \neq 0$. The trace $\operatorname{tr}$ in (10) is taken over the three-momentum and matrix indices.

From expression (10), the Ginzburg-Landau action, (2), with the coefficients (4) can be obtained by expanding in small $\Delta_{i, p}$. The mean-field value for the Cooper pair field $\Delta_{0}(T)$ is found by minimizing the action and $T_{c}$ is found by solving for the highest temperature below which the mean-field value is non-zero. In the limit $N \rightarrow \infty$, this yields the mean-field value $\Delta_{0}(0)=g p_{\mathrm{FB}}^{2} /(16 d \pi)$ and $T_{c}=\Delta_{0}(0) / 3$ which we have used in expressing the Ginzburg-Landau coefficients in terms of the critical temperature. Note that this yields a mean field $T_{c}$ that is linearly proportional to the coupling constant, contrary to the usual exponentially small $T_{c}$. More generally, we can expand $\Delta_{i}$ around this meanfield value, $\Delta_{i}=\Delta_{0}+\delta \Delta_{i}$. This separates the action into a mean-field part and a fluctuation part, $S=S_{M F}+S_{\delta}$, where the fluctuation part is given by

$$
S_{\delta}=\frac{1}{2} \sum_{q} \overrightarrow{\delta \Delta_{q}^{\dagger}}\left(\begin{array}{cccc}
-\mathcal{W}_{q}+\frac{V}{g T} & \mathcal{D}_{q} & -\mathcal{X}_{q} & 0 \\
\mathcal{D}_{q} & -\mathcal{W}_{-q}+\frac{V}{g T} & 0 & -\mathcal{X}_{-q} \\
-\mathcal{X}_{q} & 0 & -\mathcal{W}_{q}+\frac{V}{g T} & \mathcal{D}_{q} \\
0 & -\mathcal{X}_{-q} & \mathcal{D}_{q} & -\mathcal{W}_{-q}+\frac{V}{g T}
\end{array}\right) \overrightarrow{\delta \Delta}_{q}
$$

and the mean-field part by

$$
S_{M F}=-4 \sum_{\mathbf{p}} \ln \left[\cosh \left(E_{\mathbf{p}} / 2 T\right)\right]+\frac{2 V}{g T}\left|\Delta_{0}\right|^{2} .
$$

In the latter we have performed the summation over the Matsubara frequencies and $E_{\mathbf{p}}$ are the positive quasiparticle energies given by Eq. (1). We have here written the action in a convenient matrix form with the vector fluctuation fields given by $\overrightarrow{\delta \Delta}_{q}=$ $\left(\delta \Delta_{1, q}, \delta \bar{\Delta}_{1,-q}, \delta \Delta_{N, q}, \delta \bar{\Delta}_{N,-q}\right)^{T}$. The matrix elements in the fluctuation action are given in terms of three po- larization operators. Expressions for them are

$$
\begin{aligned}
\mathcal{W}_{q} & =\sum_{p} \frac{\tilde{\omega}_{p} \tilde{\omega}_{p-q}}{\left(\tilde{\omega}_{p}^{2}+\Delta_{0}^{2}+\xi_{p}^{2}\right)\left(\tilde{\omega}_{p-q}^{2}+\Delta_{0}^{2}+\xi_{p-q}^{2}\right)} \\
\mathcal{D}_{q} & =\sum_{p} \frac{\Delta_{0}^{2}}{\left(\tilde{\omega}_{p}^{2}+\Delta_{0}^{2}+\xi_{p}^{2}\right)\left(\tilde{\omega}_{p-q}^{2}+\Delta_{0}^{2}+\xi_{p-q}^{2}\right)} \\
\mathcal{X}_{q} & =\sum_{p} \frac{\xi_{p} \xi_{p-q}}{\left(\tilde{\omega}_{p}^{2}+\Delta_{0}^{2}+\xi_{p}^{2}\right)\left(\tilde{\omega}_{p-q}^{2}+\Delta_{0}^{2}+\xi_{p-q}^{2}\right)} .
\end{aligned}
$$

As for the Ginzburg-Landau action, the four eigenmodes 
of the fluctuation action correspond to relative and total phase fluctuations and relative and total amplitude fluctuations. Also, again, in the limit of a large number of layers, only the total amplitude and total phase modes survive and the third polarization operator, $\mathcal{X}_{q}$, vanishes.

From the Ginzburg-Landau calculation, we know that the dominating contribution to the thermodynamics in the large $N$ limit comes from the amplitude fluctuation part. Taking into account only this mode, the fluctuation contribution to the free energy, $\delta F=-T \ln \delta \mathcal{Z} \approx$ $\delta F_{\text {amplitude }}$, becomes

$$
\delta F_{\text {amplitude }}=2 T \sum_{q} \ln \left(1-\frac{\mathcal{W}_{q}-\mathcal{D}_{q}}{V / g T}\right)
$$

We calculate $\delta F_{\text {amplitude as }}$ well as the heat capacity, $\delta C_{\text {amplitude }}=-T \partial_{T}^{2} \delta F_{\text {amplitude, numerically. }}$ The result for the free energy is shown in Fig. 2. For zero temperature, the fluctuation contribution goes to zero, but grows larger than the mean-field contribution as the temperature increases. Above the critical temperature,

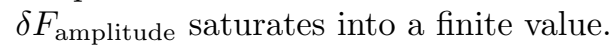

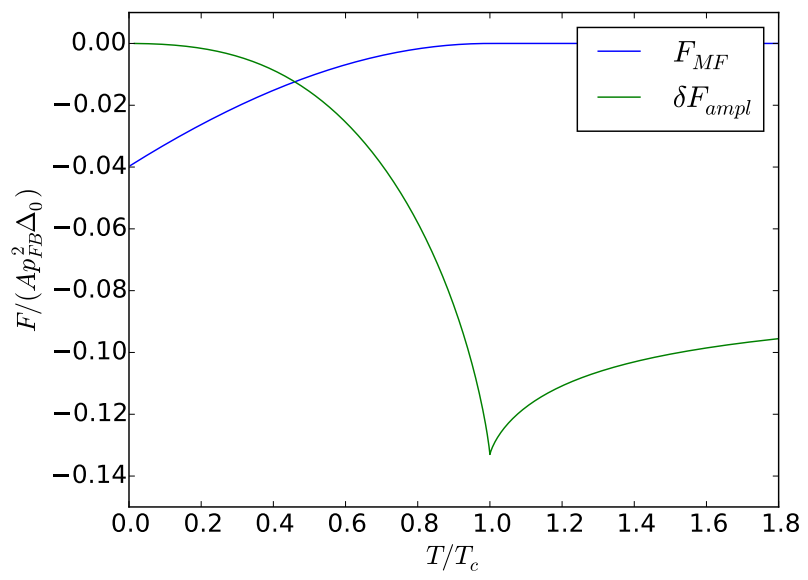

FIG. 2. (Color online) Free energy contribution from the amplitude fluctuations and from the mean-field calculation.

For the heat capacity, the numerical result is shown in Fig. 3. At low temperatures we find that the fluctuation contribution to the heat capacity becomes equal to the mean field heat capacity $C_{M F}$, both given by

$$
C\left(T \ll T_{c}\right)=\frac{A p_{\mathrm{FB}}^{2} \pi}{6} \frac{T}{\Delta_{0}} .
$$

For $C_{M F}$ Eq. (16) straightforwardly follows from Eq. (13). On the other hand, the fact that $\delta C_{\text {amplitude }}$ is also given by Eq. (16) can be analytically understood by assuming that it comes from a free boson contribution corresponding to the amplitude mode dispersion, which can be determined from the corresponding eigenvalue of the matrix in the fluctuation action (12) (the method is described for example $\left.i^{29}\right)$. Namely, this way we find

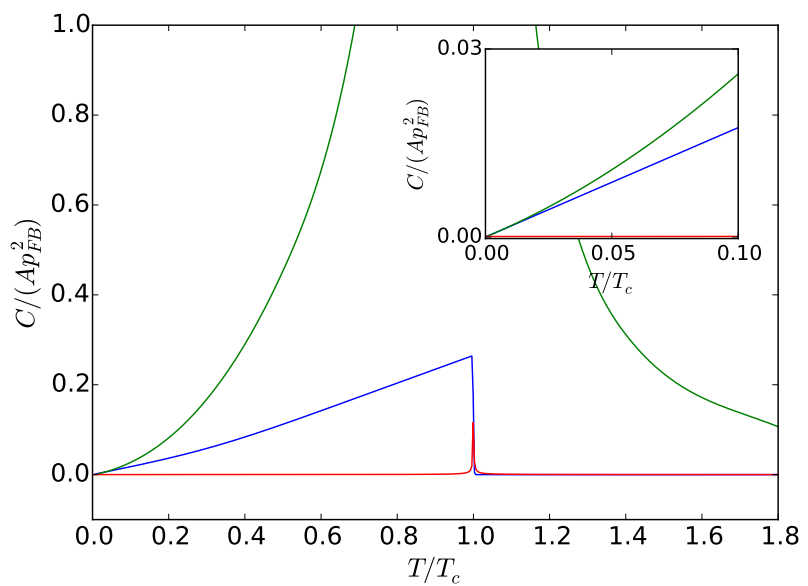

FIG. 3. (Color online) Specific heat contribution from the amplitude fluctuations (green curve) and from the meanfield calculation (blue curve). For comparison the fluctuation contribution for a conventional BCS superconductor with $T_{c}=10^{-4} E_{F}$ and equal heat capacity jump at the transition $\Delta C$ is also shown (red curve). Inset shows the lowtemperature behaviour of the three curves.

that for $T=0$ and $N \rightarrow \infty$ the amplitude mode dispersion is approximatively given by

$$
E_{\mathbf{p}, \text { ampl }}(T=0)=\Delta_{0} \mathbf{p}^{2} / p_{\mathrm{FB}}^{2}
$$

and the corresponding heat capacity is indeed given by Eq. (16). For higher temperatures, the fluctuation contribution completely dominates over the mean field contribution. At $T_{c}$, the heat capacity diverges as $\sim 1 /\left|T-T_{c}\right|$ as predicted by the Ginzburg-Landau theory. The temperature at which the fluctuation heat capacity equals the mean-field heat capacity jump at the transition is approximately $T / T_{c} \approx 0.4$ which is similar as expected from the Ginzburg number. For comparison, we also show the Ginzburg-Landau fluctuation contribution expected for a conventional BCS superconductor [Eq. (9)] with $T_{c} / E_{F} \sim 10^{-4}$ and other parameters chosen so that the mean-field heat capacity jump at the transition $\Delta C$ would be the same as the one we get for the flat band case.

The large contribution from the amplitude mode even at low temperatures can be understood from the form of the amplitude mode dispersion at $T=0$. By investigating the eigenvalues of the matrix in the fluctuation action, Eq. (12), one can show that the mass gap of the mode is not $2 \Delta_{0}$ as in conventional superconductors, but instead it is $2 E_{\mathbf{p}, \text { min }}$, where $E_{\mathbf{p}, \text { min }}$ is the minimum of the fermionic spectrum. This relation between the mass gap and $E_{\mathbf{p} \text {,min }}$ is possibly generic only to the $1 \mathrm{D}$ line of zeros within the $2 \mathrm{D}$ surface state, as opposed to the set of 0D nodal points in 3D unconventional superconductors. Since the spectrum of the fermionic excitations is given by Eq. (1), which does not have a gap in the limit of large $N$ (see Fig. 1), also the gap in the amplitude mode 
closes and the contribution from the fermionic mode and the bosonic amplitude mode can be of the same order of magnitude at low temperatures. Therefore, finite $N$ corrections to our result would likely diminish the fluctuation contribution, improving the validity range of the mean-field theory.

Since vanishing of the superconducting excitation gap along a 1D line is a general property of all topological semimetals with flat band surface state, we expect that our prediction of large fluctuation contribution and massless collective excitation spectrum applies to all materials within this class. However, this is not a general property to all superconducting states in systems exhibiting a flat normal state spectrum, as some of them may well exhibit a gapful superconducting spectrum.

Flat bands can also emerge at the surface in superconductors with a nodal order parameter structure possibly resulting in correlated states ${ }^{30-34}$. Similarly as in the present situation, the fluctuation contribution to the heat capacity may also dominate the mean field contribution in these correlated states, so that the mean field theory becomes inaccurate and the interactions lead to a strongly correlated state. In the bulk of these superconductors, the presence of the nodal points leads to a decrease of the amplitude mode masses ${ }^{39,40}$, but contrary to the superconducting topological semimetal sur- face states, this mass does not vanish.

We consider here only the effect of the amplitude mode to thermodynamics since we assume that it dominates over the phase mode contribution. At reasonably high temperatures it follows from general Ginzburg-Landau theory that the amplitude mode contribution dominates because of its singularity at $T=T_{c}$. At lower temperatures, the phase mode contribution to thermodynamic properties in a charged system is also influenced by the coupling to the electromagnetic field ${ }^{35,36}$. In the calculation of this coupling it should be taken into account that although the order parameter appears only close to the surface the supercurrent flows also in the bulk ${ }^{37}$.

Lastly, we want to emphasize that we do not consider the coupling of the fluctuations to external fields. The large contribution from the amplitude fluctuations to the heat capacity might or might not appear in other observables depending on whether they couple to this degree of freedom or not. For example, it is known that the electromagnetic field does not couple to the amplitude mode at $T=0$ directly $^{38}$ and thus such a large fluctuation effect is not necessarily present in the charge current.

We thank G.E. Volovik for helpful discussions. This work was supported by the Academy of Finland through its Center of Excellence program, and by the European Research Council (Grant No. 240362-Heattronics).
* ville.kauppila@aalto.fi

1 T. T. Heikkilä, N. B. Kopnin, and G. E. Volovik, JETP Lett. 94, 233 (2011).

2 S. Matsuura, P.-Y. Chang, A. P. Schnyder, and S. Ryu, New Journal of Physics 15, 065001 (2013).

3 F. Guinea, A. H. Castro Neto, and N. M. R. Peres, Phys. Rev. B 73, 245426 (2006).

${ }^{4}$ N. B. Kopnin, T. T. Heikkilä, and G. E. Volovik, Phys. Rev. B 83, 220503 (2011).

5 N. B. Kopnin, M. Ijäs, A. Harju, and T. T. Heikkilä, Phys. Rev. B 87, 140503 (2013).

${ }^{6}$ P. Esquinazi, T. T. Heikkilä, Y. V. Lysogorskiy, D. A. Tayurskii, and G. E. Volovik, JETP Lett. 100, 336 (2014).

7 S. Ryu and Y. Hatsugai, Phys. Rev. Lett. 89, 077002 (2002).

8 K. Mullen, B. Uchoa, and D. T. Glatzhofer, Phys. Rev. Lett. 115, 026403 (2015).

9 Y. Kim, B. J. Wieder, C. L. Kane, and A. M. Rappe, Phys. Rev. Lett. 115, 036806 (2015).

10 R. Yu, H. Weng, Z. Fang, X. Dai, and X. Hu, Phys. Rev. Lett. 115, 036807 (2015).

11 L. S. Xie, L. M. Schoop, E. M. Seibel, Q. D. Gibson, W. Xie, and R. J. Cava, arXiv preprint arXiv:1504.01731 (2015).

12 H. Weng, Y. Liang, Q. Xu, R. Yu, Z. Fang, X. Dai, and Y. Kawazoe, Phys. Rev. B 92, 045108 (2015).

13 E. Tang and L. Fu, Nature Phys. 10, 964 (2014).

14 V. A. Khodel and V. R. Shaginyan, JETP Lett. 51, 553 (1990).

15 S. Miyahara, S. Kusuta, and N. Furukawa, Physica C: Superconductivity 460, 1145 (2007).
${ }^{16}$ K. Murase, S. Ishida, S. Takaoka, T. Okumura, H. Fujiyasu, A. Ishida, and M. Aoki, Surf. Sci. 170, 486 (1986).

17 N. Y. Fogel, E. I. Buchstab, Y. V. Bomze, O. I. Yuzephovich, M. Y. Mikhailov, A. Y. Sipatov, E. A. Pashitskii, R. I. Shekhter, and M. Jonson, Phys. Rev. B 73, 161306 (2006).

18 O. Yuzephovich, M. Y. Mikhailov, S. Bengus, A. Y. Aladyshkin, E. Pestov, Y. N. Nozdrin, A. Y. Sipatov, E. Buchstab, and N. Y. Fogel, Low Temp. Phys. 34, 985 (2008).

19 T. Scheike, P. Esquinazi, A. Setzer, and W. Böhlmann, Carbon (2013).

20 T. Scheike, W. Böhlmann, P. Esquinazi, J. BarzolaQuiquia, A. Ballestar, and A. Setzer, Adv. Mat. 24, 5826 (2012).

21 A. Ballestar, J. Barzola-Quiquia, T. Scheike, and P. Esquinazi, New J. Phys. 15, 023024 (2013).

22 V. I. Iglovikov, F. Hébert, B. Grémaud, G. G. Batrouni, and R. T. Scalettar, Phys. Rev. B 90, 094506 (2014).

23 J. E. Hirsch and D. J. Scalapino, Phys. Rev. Lett. 56, 2732 (1986).

24 W. A. Muñoz, L. Covaci, and F. M. Peeters, Phys. Rev. B 87, 134509 (2013).

${ }^{25}$ We use $|\mathbf{p}|=(1-1 / N) p_{F B}$ as a cut-off momentum in all integrals.

${ }^{26}$ We use term Cooper pair field for the fluctuating complex fields $\Delta_{1, N}$ in the action. The corresponding mean field is the pair potential.

27 A. Larkin and A. Varlamov, Theory of Fluctuations in Superconductors, International Series of Monographs on Physics (OUP Oxford, 2005).

${ }^{28}$ In addition to the GL coefficients the value of the heat 
capacity depends also on the choice of the momentum cutoff $^{25}$.

${ }^{29}$ V. Popov, Functional Integrals and Collective Excitations, Cambridge Monographs on Mathematical Physics (Cambridge University Press, 1987).

30 A. P. Schnyder, P. M. R. Brydon, and C. Timm, Phys. Rev. B 85, 024522 (2012).

31 C. Honerkamp, K. Wakabayashi, and M. Sigrist, EPL 50, 368 (2000).

${ }^{32}$ M. Covington, M. Aprili, E. Paraoanu, L. H. Greene, F. Xu, J. Zhu, and C. A. Mirkin, Phys. Rev. Lett. 79, 277 (1997).
33 A. C. Potter and P. A. Lee, Phys. Rev. Lett. 112, 117002 (2014).

34 Y. Li, D. Wang, and C. Wu, New J. Phys. 15, 085002 (2013).

35 P. W. Anderson, Phys. Rev. 112, 1900 (1958).

36 P. W. Anderson, Phys. Rev. 130, 439 (1963).

37 N. B. Kopnin, JETP Lett. 94, 81 (2011).

38 P. B. Littlewood and C. M. Varma, Phys. Rev. B 26, 4883 (1982).

39 Y. Barlas and C.M. Varma, Phys. Rev. B 87, 054503 (2013).

40 G.E. Volovik and M.A. Zubkov, J. Low Temp. Phys. 175, 486 (2014). 